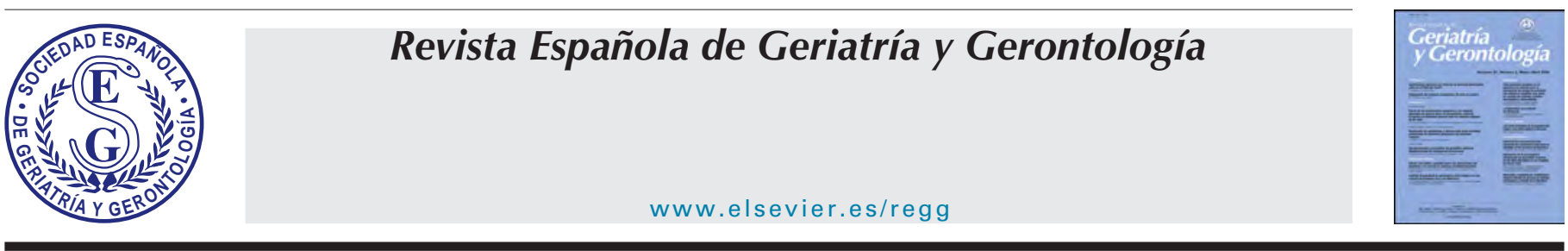

ORIGINAL

\title{
Influencia de la ocupación en el deterioro cognitivo libre de demencia en una muestra de sujetos mayores de 55 años de Zaragoza
}

\author{
Ana Cristina Gracia-Rebled ${ }^{\mathrm{a}, *}$, Javier Santabárbara ${ }^{\mathrm{b}, \mathrm{c}}$, Raul Lopez-Anton ${ }^{\mathrm{c}, \mathrm{d}}$, Concepción Tomas ${ }^{\mathrm{a}}$, \\ Elena Lobo ${ }^{\mathrm{b}, \mathrm{c}}$, Guillermo Marcos ${ }^{\mathrm{b}, \mathrm{c}, \mathrm{e}}$ y Antonio Lobo ${ }^{\mathrm{c}, \mathrm{f,g}}$ \\ a Departamento de Fisiatría y Enfermería, Universidad de Zaragoza, Zaragoza, España \\ b Departamento de Microbiología, Medicina Preventiva y Salud Pública, Universidad de Zaragoza, Zaragoza, España \\ c Instituto Universitario de Investigación Sanitaria de Aragón (IIS) \\ d Departamento de Psicología y Sociología, Universidad de Zaragoza, Zaragoza, España \\ e Hospital Clínico Universitario, Zaragoza, España \\ ${ }^{\mathrm{f}}$ Departamento de Medicina, Psiquiatría y Dermatología, Universidad de Zaragoza, Zaragoza, España \\ g Centro de Investigación Biomédica en Red de Salud Mental (CIBERSAM), Ministerio de Ciencia e Innovación, Madrid, España
}

\section{INFORMACIÓN DEL ARTÍCULO}

\section{Historia del artículo:}

Recibido el 19 de enero de 2017

Aceptado el 14 de julio de 2017

On-line el xxx

\section{Palabras clave:}

Ocupación

Deterioro cognitivo

Salud laboral

Salud mental

Análisis de regresión logística

\begin{abstract}
R E S U M E N
Introducción: La prevalencia de deterioro cognitivo libre de demencia (DCLD) varía entre el 5,1 y el 35,9\%, con un aumento entre los 65 y los 85 años. El DCLD incrementa el riesgo de demencia. Factores como nivel educativo, ocupación y actividades sociales se asocian con el riesgo de deterioro cognitivo. El objetivo principal de este estudio fue analizar la asociación entre la principal ocupación a lo largo de la vida y el DCLD en una muestra de población general de mayores de 55 años.

Material y métodos: En la fase I del proyecto ZARADEMP se entrevistó a una muestra $(\mathrm{n}=4.803)$ de personas mayores de 55 años. La medida del DCLD se obtuvo a través del Examen Cognoscitivo Mini Mental. La actividad laboral se recodificó en cuello blanco, cuello azul, labores del hogar y agricultores. Se estimó la asociación entre las variables ocupación y DCLD a través de odds ratio e intervalos de confianza del 95\% mediante ecuaciones de regresión logística.

Resultados: La prevalencia de DCLD en la muestra fue del $28,2 \%$. Con respecto a los trabajadores de cuello blanco, la odds ratio de diagnóstico de DCLD fue un 53\% mayor para aquellos trabajadores de cuello azul, un $77 \%$ mayor para mujeres que desempeñaron labores del hogar y casi el doble para los agricultores, tras controlar por variables sociodemográficas, conductuales y clínicas. Todos los resultados fueron estadísticamente significativos.

Conclusiones: La frecuencia de DCLD está influida por la ocupación laboral previa de los sujetos. Una ocupación con mayores requerimientos intelectuales puede ayudar a mantener las funciones cognitivas intactas durante más tiempo.
\end{abstract}

(c) 2017 SEGG. Publicado por Elsevier España, S.L.U. Todos los derechos reservados.

\section{Influence of occupation on cognitive impairment with no dementia in a sample population over 55 years from Zaragoza}

\begin{abstract}
A B S T R A C T
Introduction: The prevalence of cognitive impairment with no dementia (CIND) varies between $5.1 \%$ and $35.9 \%$, increasing between 65 and 85 years. The CIND increases the risk of dementia. Factors such as education, occupation, and social activities are associated with the risk of cognitive impairment. The main objective of this study was to analyse the association between the main occupation developed throughout life and CIND in a general population sample of over 55 years.

Methods: In wave I of the ZARADEMP Project, a sample $(\mathrm{n}=4803)$ of people over 55 years was interviewed. CIND measurement was obtained through the Mini Mental State Examination. Occupational activity data were recoded into white collar, blue collar, homemakers, and farmers. The association between the
\end{abstract}

\footnotetext{
* Autor para correspondencia.

Correo electrónico: acgracia@unizar.es (A.C. Gracia-Rebled).
} 
occupation variables and CIND was estimated using the odds ratio, and 95\% confidence intervals using logistic regression equations.

Results: The prevalence of CIND in the sample was $28.2 \%$. As regards white collar workers, the CIND diagnosis odds was $53 \%$ higher for blue collar workers, $77 \%$ higher for women who were homemakers and almost twice for farmers, after controlling for socio-demographic, behavioural and clinical variables. All results were statistically significant.

Conclusions: CIND frequency is influenced by the previous occupation of the subjects. An occupation with higher intellectual requirements can help keep cognitive functions intact for longer.

@ 2017 SEGG. Published by Elsevier España, S.L.U. All rights reserved.

\section{Introducción}

El deterioro cognitivo libre de demencia (DCLD) se define por una disminución del rendimiento cognitivo que no puede ser considerado normal para la edad y nivel de educación de los sujetos, pero que no alcanza la intensidad suficiente como para llegar al diagnóstico de demencia ${ }^{1}$. Su prevalencia varía según los estudios y la definición utilizada, y se sitúa entre el 5,1 y el $35,9 \%^{2-4}$, con un aumento de prevalencia con la edad entre los 65 y los 85 años. El DCLD incrementa en casi 3 veces el riesgo de demencia a los 3 años de seguimiento con respecto a las personas con cognición normal ${ }^{5,6}$.

Conocer los factores de riesgo que se asocian al DCLD puede ayudar a retrasar su aparición. En este sentido, se han identificado algunos factores no modificables, como edad o sexo, y otros potencialmente modificables, como los asociados al estilo de vida o los conductuales como consumo de tabaco, alcohol y sobrepeso/obesidad y variables clínicas tales como hipertensión, diabetes, enfermedad vascular y depresión ${ }^{3,5,7}$.

Además, la bibliografía recoge que diversos factores entre los que se encuentran el nivel educativo, la complejidad ocupacional y el compromiso social valorado según el contacto con familiares y vecinos, así como la asistencia a eventos sociales, se asocian con el riesgo de padecer deterioro cognitivo ${ }^{8}$. Estudiamos la ocupación porque se considera que aquellos trabajos en los que se exige mayor rendimiento intelectual pueden proteger frente a problemas $\operatorname{cognitivos}^{9-11}$. Estudios transversales que analizan la ocupación en relación con el deterioro cognitivo concluyen que los trabajadores de cuello azul tienen menores puntuaciones medias en tests cognitivos que los trabajadores de cuello blanco ${ }^{9}$, con diferencias cognitivas según la ocupación de los sujetos ${ }^{10}$. Aunque algunos autores piensan que el rendimiento cognitivo está influido por la educación y no por la ocupación ${ }^{12}$.

La mayoría de las publicaciones relacionan la ocupación laboral con el desarrollo de demencia10,13-19, enfermedad de Alzheimer ${ }^{15,16,19-21}$ o deterioro cognitivo leve $e^{8-11,22-25}$. Sin embargo, son menos los trabajos que relacionan la ocupación laboral con el DCLD ${ }^{7}$.

Un estudio más exhaustivo de las características de la ocupación que influyen en el incremento de la frecuencia de DCLD podría mejorar el conocimiento de la asociación entre ambas variables, o podría ayudar a elaborar programas de prevención primaria en aquellos individuos que, dada su ocupación, tienen mayor probabilidad de padecer DCLD.

Por ello, el objetivo principal de este estudio fue analizar la asociación entre la principal ocupación desarrollada por los sujetos a lo largo de la vida y el DCLD en una muestra de población general de personas mayores de 55 años.

\section{Sujetos y métodos}

\section{Diseño del estudio}

El estudio ZARADEMP es un estudio longitudinal, diseñado para documentar la prevalencia de demencia y depresión en la población de 55 o más años de la ciudad de Zaragoza ${ }^{26}$. Se utilizó un diseño epidemiológico de detección de casos de demencia en 2 fases, llevado a cabo entre los años 1994 y 1996.

El Comité de Ética de la Universidad de Zaragoza y el Fondo de Investigación Sanitaria aprobaron el proyecto de acuerdo con la legislación española vigente y todos los individuos dieron su consentimiento informado por escrito; se mantuvieron la intimidad y la confidencialidad a lo largo del proyecto.

\section{Muestra del estudio}

Inicialmente, se seleccionó una muestra aleatoria, estratificada por edad y sexo, de personas mayores de 55 años, tomando como referencia la información oficial del padrón municipal de residentes de la ciudad de Zaragoza en el censo del año 1991, incluyendo tanto a personas institucionalizadas como a no institucionalizadas.

En la entrevista inicial, fase I, entrevistadores bien entrenados y supervisados regularmente realizaron la entrevista ZARADEMP (entre 25 y 90 min de duración) en el lugar de residencia de los sujetos. La muestra inicial de personas entrevistadas ascendió a 4.803.

En la fase II, psiquiatras investigadores del equipo reevaluaron, usando los mismos instrumentos que en la fase I y que se detallan en el siguiente apartado, a aquellos individuos que se consideraban "probables casos de demencia» (especialmente a las personas con dificultades cognitivas), en ocasiones, en presencia de los cuidadores cuando al entrevistado se lo consideraba poco fiable. Al final de la fase II, un panel de psiquiatras diagnosticó los casos de demencia utilizando los criterios del DSM-IV-TR. Nuestros estudios anteriores apoyan la validez de este procedimiento ${ }^{27}$ en la detección de casos. Para este estudio, los casos identificados de demencia $(n=223)$ fueron excluidos y quedó una muestra de 4.580 sujetos para el análisis.

\section{Variables de estudio e instrumentos}

Todas las variables empleadas para este estudio fueron codificadas a partir de la información recogida en la entrevista ZARADEMP, que incluye versiones estandarizadas en español de la Geriatric Mental State-History and Aetiology Schedule-Automated Geriatric Examination for Computer Assisted Taxonomy package (GMS-HASAGECAT $)^{28}$ y del European Community Concerted Action on the Epidemiology and Prevention of Dementia (EURODEM) Risk Factors Questionnaire ${ }^{29}$, con información relacionada con la historia médica y psiquiátrica. El diseño y principales objetivos de ZARADEMP han sido publicados con antelación ${ }^{26}$.

\section{Deterioro cognitivo libre de demencia}

La medida del DCLD se obtuvo a través del Examen Cognoscitivo Mini Mental (ECMM) ${ }^{30}$, versión española estandarizada del MiniMental Status Examination de Folstein (MMSE) ${ }^{31}$ como un indicador fiable y válido de la función cognitiva.

Los grados de deterioro cognitivo basados en las puntuaciones del MMSE validados por Perneczky et al. ${ }^{32}$ son: "normal» (30), «cuestionable» (puntuaciones 26-29), «leve» (puntuaciones 
21-25), «moderado» (11-20) y "grave» (puntuaciones 0-10). Para los propósitos del presente trabajo, el punto de corte del ECMM para detectar un caso de DCLD fue establecido en 25/26 puntos.

\section{Ocupación}

La ocupación se consideró como la principal actividad que realizaron los sujetos a lo largo de su vida, ya fuera esta remunerada o no. La entrevista ZARADEMP recogió datos de la actividad laboral y se clasificaron en 18 grupos (Anexo 1). Para la codificación posterior se tuvieron en cuenta tanto la Clasificación Nacional de Ocupaciones como la Clasificación Internacional Uniforme de Ocupaciones, utilizamos las versiones actualizadas de 2011 (CNO-11) (CN $^{33}$ y de 2008 (CIUO-08) (34 $^{3}$, lo que posibilitó la comparación con otros estudios nacionales e internacionales (Anexo 2).

A su vez, siguiendo el modelo del Estudio Toledo de Envejecimiento Saludable ${ }^{15}$ se agruparon en el nivel cuello blanco los grupos 1 a 5 del CNO-11, excepto los servicios de protección. En el nivel de cuello azul se incluyeron los grupos 7, 8, parte del 9 y los servicios de protección y militares. El nivel correspondiente a labores del hogar formó parte del grupo 9 (01 y 10 de la entrevista). Por último, dentro de la categoría agricultores se incluyeron el grupo 6 de la CNO-11 y el grupo 04 de la entrevista, incluido dentro de las ocupaciones elementales de la CNO-11 (Anexo 3).

Posteriormente, para su posible comparación con otros estudios, se agruparon estas 4 categorías en 2 : manual, donde se incluyeron los trabajadores de cuello azul, labores del hogar y agricultores, e intelectual, con los trabajadores de cuello blanco.

\section{Otras covariables}

Otros potenciales factores de confusión fueron evaluados al inicio del estudio e incluían variables sociodemográficas como: edad, sexo y escolarización; estilos de vida: consumo de alcohol, tabaco, e índice de masa corporal (IMC) y factores de riesgo cardiovascular: hipertensión, diabetes y enfermedad vascular y depresión.

La escolarización se categorizó en 3 niveles: analfabeto, que no saben leer y escribir, o menos de 2 años de escolarización formal; primaria completa o incompleta y estudios medios o superiores.

El consumo de alcohol en el momento de la entrevista, agrupado en abstemios, que incluían los consumidores muy ocasionales de muy pequeñas cantidades de alcohol; exbebedores y bebedores habituales.

El consumo de tabaco fue registrado como no fumador; exfumador; fumador.

El IMC se calculó como el peso en kilogramos dividido por la altura en metros al cuadrado y se clasificó como normopeso entre 20 y $24,99 \mathrm{~kg} / \mathrm{m}^{2}$, sobrepeso entre 25 y $29,99 \mathrm{~kg} / \mathrm{m}^{2}$ y obesidad un IMC $^{35}$ igual o superior a $30 \mathrm{~kg} / \mathrm{m}^{2}$.

La presión arterial (PA) se midió durante la realización de la entrevista mediante un tensiómetro manual estándar, utilizando el promedio de 2 lecturas; se consideró hipertensión cuando PA > 140/90 mmHg o si el participante informó que estaba siendo tratado de hipertensión, lo que fue verificado por el entrevistador por los fármacos que estaba consumiendo.

La presencia de factores de riesgo vascular y diabetes se basó en la historia médica obtenida utilizando el Cuestionario de Factores de Riesgo EURODEM ${ }^{29}$ y los fármacos consumidos.

La historia de enfermedad vascular se consideró como variable dicotómica, distinguiendo entre presencia y ausencia de antecedentes personales.

La diabetes también se consideró como una variable dicotómica que distingue entre personas con un diagnóstico previo o un tratamiento médico para la diabetes y ausencia de antecedentes de diabetes.
Para el diagnóstico de "caso de depresión» se utilizó el sistema computarizado AGECAT, el cual emite un diagnóstico jerárquico a partir de los datos de la entrevista recogidos con el instrumento GMS.

\section{Análisis estadístico}

La comparación de proporciones de las variables cualitativas según la categoría laboral se realizó mediante el contraste de chicuadrado y la comparación de medias en las variables continuas se realizó mediante el contraste ANOVA.

En un primer paso del análisis de regresión, una vez excluidos los sujetos diagnosticados de demencia, se estimó la asociación transversal entre las variables ocupación y DCLD al inicio del estudio a través de la odds ratio (OR) y sus intervalos de confianza del 95\% (IC) calculados mediante ecuaciones de regresión logística. Con el fin de explorar los mecanismos que explican la asociación, se utilizaron 3 modelos en los que se controlaron gradualmente posibles factores de confusión/modificadores del efecto. El modelo 1 incluyó el estatus ocupacional y las características sociodemográficas (edad, sexo y escolarización). El modelo 2 incluyó los términos del modelo 1 más los factores de riesgo conductuales (consumo de tabaco, consumo de alcohol y obesidad), factores de riesgo clínico (hipertensión, diabetes y enfermedad vascular) y condiciones psiquiátricas (depresión). Se evaluaron las interacciones entre las covariables y el estatus ocupacional antes de calcular el modelo de regresión multivariante, y no se encontró significación estadística en ningún caso. En el modelo 3 se eliminó la categoría laboral para ver en qué medida afectaba a la significación del resto de las variables y al valor predictivo del modelo.

En un segundo paso del análisis de regresión, se estudió la asociación entre el tipo de actividad laboral (manual vs. intelectual) y el DCLD ajustando por las mismas covariables que en el paso anterior.

Se evaluó la bondad del ajuste de cada uno de los modelos mediante el contraste de Hosmer-Lemeshow ${ }^{36}$, exigiendo un valor $p>0,05$.

El porcentaje de variabilidad del diagnóstico explicada por las variables independientes de cada modelo de regresión fue evaluado mediante el coeficiente de determinación de Nagelkerke ${ }^{37}$.

El análisis de los datos fue llevado a cabo con el paquete estadístico SPSS v19 (IBM Corp, NY, EE. UU.).

\section{Resultados}

De los 4.580 sujetos libres de demencia que componían la muestra inicial, 4.389 sujetos (91,3\%) fueron clasificados según la ocupación laboral principal desarrollada a lo largo de la vida. De ellos, 1.001 (22,8\%) individuos pertenecían a la categoría de cuello blanco, $1.354(30,8 \%)$ a la de cuello azul, $1.665(37,9 \%)$ mujeres se dedicaban a labores del hogar y $369(8,4 \%)$ eran agricultores.

La tabla 1 muestra la distribución de las características sociodemográficas, de estilo de vida y salud de los sujetos de la muestra según la categoría laboral desarrollada. Entre los agricultores se encontró un menor porcentaje de mujeres, así como un mayor porcentaje de sujetos mayores de 80 años y analfabetos. Las mujeres que desempeñaban labores del hogar tuvieron menor frecuencia de consumo de tabaco y alcohol y de padecer enfermedad cardiovascular, así como una mayor frecuencia de padecer obesidad, hipertensión, diabetes y depresión.

Con relación a la evaluación de las puntuaciones cognitivas recogidas con el ECMM, de los 4.389 sujetos clasificados según la ocupación laboral principal desarrollada a lo largo de la vida se observó que $1.238(28,2 \%)$ tenían resultados compatibles con DCLD. De los sujetos con ocupación de cuello blanco, 120 (12,0\%) obtuvieron puntuaciones por debajo del punto de corte; en el grupo de 
Tabla 1

Distribución de los sujetos de la muestra según la categoría laboral desarrollada

\begin{tabular}{|c|c|c|c|c|c|}
\hline & Cuello blanco $(\mathrm{n}=1.001)$ & Cuello azul $(\mathrm{n}=1.354)$ & Labores del hogar $(n=1.665)$ & Agricultores $(n=369)$ & $\mathrm{p}$ \\
\hline Sexo & & & & & $<0,001$ \\
\hline Hombres, n (\%) & $682(68,1)$ & $900(66,5)$ & 0 & $280(75,9)$ & \\
\hline Mujeres, n (\%) & $319(31,9)$ & $454(33,5)$ & $1.665(100)$ & $89(24,1)$ & \\
\hline Edad media $(D E)$ & $71,4(9,2)$ & $72,4(9,3)$ & $73,5(9,6)$ & $76,9(9,5)$ & $<0,001$ \\
\hline$\leq 64, \mathrm{n}(\%)$ & $288(28,8)$ & $346(25,6)$ & $356(21,4)$ & $40(10,8)$ & \\
\hline $65-79, \mathrm{n}(\%)$ & $500(50,0)$ & $662(48,9)$ & $844(50,7)$ & $184(49,9)$ & \\
\hline$\geq 80, \mathrm{n}(\%)$ & $213(21,3)$ & $346(25,6)$ & $465(27,9)$ & $145(39,3)$ & \\
\hline Años de estudio, media (DE) & $10,1(4,7)$ & $6,5(2,9)$ & $6,1(3,0)$ & $5,2(2,8)$ & $<0,001$ \\
\hline Analfabeto, n (\%) & $23(2,3)$ & $124(9,2)$ & $236(14,3)$ & $74(20,2)$ & \\
\hline Primarios, n (\%) & $549(55,3)$ & $1.112(82,7)$ & $1.303(78,9)$ & $283(77,3)$ & \\
\hline Medios o superiores, n (\%) & $421(42,4)$ & $109(8,1)$ & $112(6,8)$ & $9(2,5)$ & \\
\hline Consumo de alcohol & & & & & $<0,001$ \\
\hline Abstemio, n (\%) & $487(52,0)$ & $652(51,5)$ & $1.441(89,7)$ & $154(44,3)$ & \\
\hline Exbebedor, n (\%) & $122(13,0)$ & $243(19,2)$ & $65(4,0)$ & $101(29,0)$ & \\
\hline Consumidor, n (\%) & $327(34,9)$ & $372(9,4)$ & $100(6,2)$ & $93(26,7)$ & \\
\hline Consumo de tabaco & & & & & $<0,001$ \\
\hline No fumador, n (\%) & $451(52,0)$ & $653(55,3)$ & $1.571(95,2)$ & $207(64,1)$ & \\
\hline Exfumador, n(\%) & $220(25,4)$ & $267(22,6)$ & $36(2,2)$ & $68(21,1)$ & \\
\hline Fumador, n (\%) & $196(22,6)$ & $261(22,1)$ & $43(2,6)$ & $48(14,9)$ & \\
\hline Índice de masa corporal & & & & & $<0,001$ \\
\hline Normopeso, n (\%) & $373(37,4)$ & $404(29,8)$ & $461(27,9)$ & $100(27,1)$ & \\
\hline Sobrepeso, n (\%) & $517(51,9)$ & $741(54,7)$ & $901(54,5)$ & $215(58,3)$ & \\
\hline Obesidad, n (\%) & $107(10,7)$ & $209(15,4)$ & $292(17,7)$ & $54(14,6)$ & \\
\hline Hipertensión arterial, $n$ (\%) & $650(65,1)$ & $918(67,9)$ & $1.189(71,4)$ & $249(67,7)$ & 0,029 \\
\hline Diabetes, $n(\%)$ & $105(10,5)$ & $186(13,9)$ & $236(14,3)$ & $38(10,4)$ & 0,014 \\
\hline Historia de enfermedad cardiovascular, $n(\%)$ & $46(4,8)$ & $102(7,9)$ & $45(2,8)$ & $27(7,8)$ & $<0,001$ \\
\hline Depresión, $n$ (\%) & $82(8,2)$ & $142(10,5)$ & $281(16,9)$ & $27(7,3)$ & $<0,001$ \\
\hline Puntuación en el ECMM, media (DE) & $27,8(2,7)$ & $26,5(3,4)$ & $25,7(3,9)$ & $25,0(4,4)$ & $<0,001$ \\
\hline Deterioro cognitivo según el ECMM, $n(\%)$ & $120(12,0)$ & $349(25,8)$ & $605(36,3)$ & $164(44,4)$ & $<0,001$ \\
\hline
\end{tabular}

trabajadores de cuello azul, fueron 349 (25,8\%) los que puntuaron bajo; las mujeres que se habían dedicado a labores del hogar con DCLD fueron 605 (36,3\%) y en la categoría de los agricultores, 164 $(44,4 \%)$.

\section{Regresión logística binaria}

En un primer paso, se estimó la asociación entre la categoría laboral y el deterioro cognitivo en la muestra de sujetos libres de demencia a través de OR y sus IC del 95\% calculados mediante ecuaciones de regresión logística.

En el modelo 1 (tabla 2) se observó una asociación estadísticamente significativa entre la categoría laboral y el diagnóstico de DCLD $(p<0,001)$, una vez eliminado el efecto de las características sociodemográficas. Así, con respecto a la categoría de trabajadores de cuello blanco, la odds de diagnóstico de DCLD fue un $61 \%$ mayor para aquellos trabajadores de cuello azul, un $91 \%$ mayor para aquellas mujeres que desempeñaron labores del hogar y 2,6 veces mayor para los agricultores. Una vez ajustado por el resto de potenciales y variables de confusión o modificadores del efecto (modelo 2), la magnitud de la asociación entre la categoría laboral y el DCLD persistió. En el modelo 3 (tabla 2), se eliminó la variable categoría laboral y se observó un leve descenso del valor predictivo del modelo, con un $\mathrm{R}^{2}$ de Nagelkerke inferior al del modelo con ocupación. Se observó que la significación de la variable de consumo de alcohol desapareció en el modelo que incluye la categoría laboral.

En un segundo paso, se estimó que aquellos que desempeñaron actividades manuales tuvieron una probabilidad de DCLD un $80 \%$ mayor que aquellos que desempeñaron actividades intelectuales.

\section{Discusión}

En este estudio, los análisis revelaron que existe asociación estadísticamente significativa entre la principal ocupación desarrollada y el DCLD.

Esta relación persistió incluso después de controlar por variables sociodemográficas y antecedentes personales, tales como el sexo, la edad, escolarización, hipertensión, diabetes, enfermedad cardiovascular, el consumo de alcohol y de tabaco, obesidad y diagnóstico de depresión. Tal como se preveía, los individuos con ocupaciones manuales obtuvieron peores resultados y, por tanto, presentaron mayor frecuencia de deterioro cognitivo que aquellos que se habían dedicado a actividades con mayor requerimiento intelectual; la frecuencia fue superior para los que habían realizado tareas agrícolas en comparación con aquellos que se habían dedicado a actividades categorizadas como de cuello blanco.

Los resultados encontrados por Cimadevila en Santiago de Compostela ${ }^{11}$ ponen de manifiesto que los agricultores y las mujeres dedicadas a labores domésticas presentan un porcentaje mayor de sujetos con síntomas de deterioro, el 41,0 y el 32,7\% respectivamente, frente al 10,9 y el $13,6 \%$ de los sujetos de profesiones de cuadros superiores o medios, proporciones superiores a las encontradas en nuestro estudio, quizá porque en el estudio gallego no eliminaron a los sujetos con diagnóstico de demencia: concluyeron que profesión y deterioro cognitivo, en el MMSE, están relacionadas.

En el estudio Paquid ${ }^{9}$, el riesgo de deterioro cognitivo fue 6 veces mayor en los trabajadores agrícolas que en el resto de las profesiones $(\mathrm{OR}=6,1$ con respecto a ocupaciones intelectuales; IC 95\%: 3,3-11,4), mayor que el hallado en nuestro estudio.

Una limitación de este trabajo para su extrapolación al momento actual, aunque no a sus conclusiones, puede ser que los datos recogidos en las entrevistas datan de principios de los años 90 , por lo 
Tabla 2

Asociación entre DCLD y categoría laboral. Modelos de regresión logística binaria

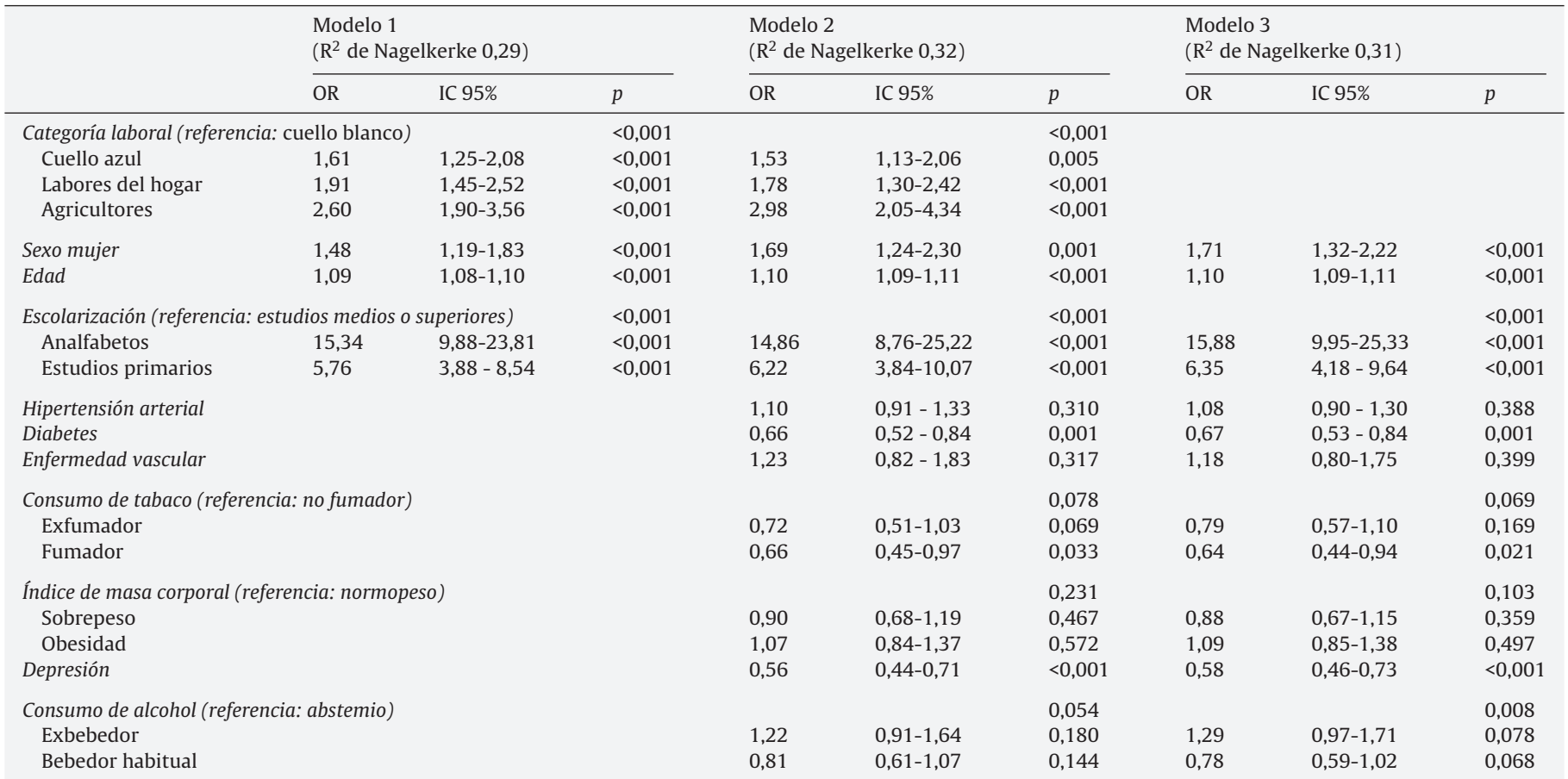

Modelo 1: categoría laboral, sexo, edad, escolarización.

Modelo 2: modelo 1+ hipertensión, diabetes, enfermedad cardiovascular, consumo de tabaco, índice de masa corporal, depresión y consumo de alcohol.

Modelo 3: sexo, edad, escolarización, hipertensión, diabetes, enfermedad cardiovascular, consumo de tabaco, índice de masa corporal, depresión y consumo de alcohol.

que las características de la muestra seleccionada son diferentes de las de la población actual de Zaragoza. No obstante, este estudio es necesario como punto de partida para poder realizar un análisis longitudinal. Otra limitación radica en que los datos recogidos de las ocupaciones no se corresponden con las categorías de la actual Clasificación Nacional de Ocupaciones y ha sido necesario hacer las pertinentes conversiones y agrupaciones para adaptarlos, por lo que puede darse algún sesgo en la clasificación de los sujetos.

En la heterogeneidad del diseño de los estudios publicados y de la clasificación de las ocupaciones desarrolladas por los sujetos, radica una de las fortalezas de nuestro estudio, ya que para codificar las categorías laborales nos hemos basado en instrumentos estandarizados como la CNO-11 y la CIUO-08. De esta manera se posibilita la comparación con otros estudios nacionales e internacionales, respectivamente.

En otras variables, como la escolarización, también se puede sospechar que se ha producido un cambio en las características del censo actual, al reducirse considerablemente la proporción de personas sin estudios en todos los grupos de edad y sexo. El rendimiento en el ECMM se afecta por la escolarización y, puesto que los agricultores tienen significativa menor escolarización en esta muestra, esto puede haber afectado a los resultados, a pesar de haber controlado por esta variable en los análisis de regresión. En el momento del diseño del estudio, el ECMM era el instrumento más adecuado para el cribado de deterioro cognitivo y, a juzgar por su uso internacional hasta el momento actual, sigue siendo un instrumento útil y válido para comparaciones internacionales. No obstante, el hecho de no disponer de una medida alternativa de DCLD puede ser considerado una limitación en el contexto actual.

Como conclusión, los resultados obtenidos avalan la hipótesis inicial de que la frecuencia de DCLD está influida por el tipo de ocupación laboral previa de los sujetos. Una ocupación con mayores requerimientos intelectuales puede ayudar a que se favorezca el mantenimiento de las funciones cognitivas intactas durante más tiempo y retrasar el inicio de la enfermedad. Estos resultados podrían servir como base sobre la que desarrollar futuros estudios longitudinales destinados a medir la relación entre la ocupación laboral desarrollada y el deterioro cognitivo a lo largo del tiempo en adultos mayores de 55 años y para el diseño de intervenciones preventivas a nivel poblacional.

\section{Conflicto de intereses}

A. Lobo recibió honorarios o ayuda de viaje de Lundbeck, aunque esta actividad no está relacionada con el proyecto actual. Para los autores restantes, no se declararon conflictos de intereses.

\section{Agradecimientos}

Los autores reconocen la contribución de los entrevistadores legos, estudiantes de medicina y miembros del grupo de trabajo ZARADEMP que participaron en el estudio.

El proyecto contó con el apoyo de donaciones del Fondo de Investigación Sanitaria del Instituto de Salud Carlos III, del Ministerio de Economía y Competitividad de Madrid (Becas 94/1562, 97/1321E, 98/0103, 01/0255, 03/0815, 06/0617, G03/128), del Gobierno de Aragón y del Fondo Social Europeo.

\section{Appendix A. Anexo 1}

Categorías laborales recogidas en la entrevista 


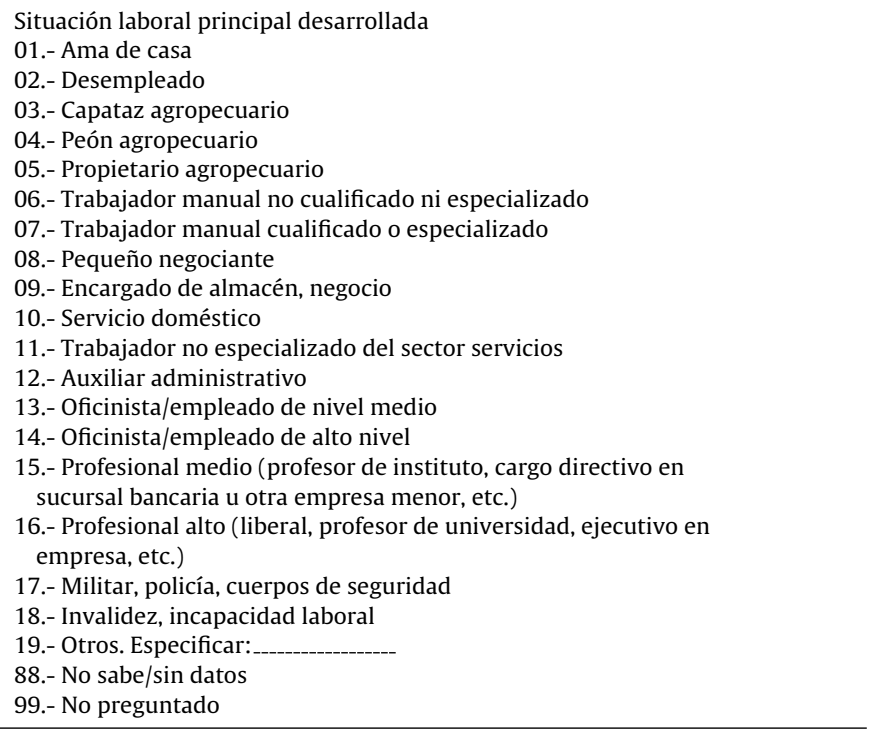

\section{Appendix B. Anexo 2}

Correspondencia entre códigos CIUO-08, CNO-11 y los utilizados en la entrevista ZARADEMP

\begin{tabular}{|c|c|c|c|}
\hline & CIUO-08 & CNO-11 & ZARADEMP \\
\hline 1 & Directores y gerentes & Directores y gerentes & 16 \\
\hline 2 & $\begin{array}{l}\text { Profesionales científicos e } \\
\text { intelectuales }\end{array}$ & $\begin{array}{l}\text { Técnicos y profesionales } \\
\text { científicos e intelectuales }\end{array}$ & 14,15 \\
\hline 3 & $\begin{array}{l}\text { Técnicos y profesionales de } \\
\text { nivel medio }\end{array}$ & $\begin{array}{l}\text { Técnicos; profesionales de } \\
\text { apoyo }\end{array}$ & 13 \\
\hline 4 & $\begin{array}{l}\text { Personal de apoyo } \\
\text { administrativo }\end{array}$ & $\begin{array}{l}\text { Empleados contables, } \\
\text { administrativos y otros } \\
\text { empleados de oficina }\end{array}$ & 12 \\
\hline 5 & $\begin{array}{l}\text { Trabajadores de los } \\
\text { servicios y vendedores de } \\
\text { comercios y mercados }\end{array}$ & $\begin{array}{l}\text { Trabajadores de los } \\
\text { servicios de restauración, } \\
\text { personales, protección y } \\
\text { vendedores }\end{array}$ & $08,09,11$ \\
\hline 6 & $\begin{array}{l}\text { Agricultores y trabajadores } \\
\text { calificados agropecuarios, } \\
\text { forestales y pesqueros }\end{array}$ & $\begin{array}{l}\text { Trabajadores cualificados } \\
\text { en el sector agrícola, } \\
\text { ganadero, forestal y } \\
\text { pesquero }\end{array}$ & 03,05 \\
\hline 7 & $\begin{array}{l}\text { Oficiales, operarios y } \\
\text { artesanos de artes } \\
\text { mecánicas y de otros } \\
\text { oficios }\end{array}$ & $\begin{array}{l}\text { Artesanos y trabajadores } \\
\text { cualificados de las } \\
\text { industrias manufactureras } \\
\text { y la construcción (excepto } \\
\text { operadores de } \\
\text { instalaciones y maquinaria) }\end{array}$ & 07 \\
\hline 8 & $\begin{array}{l}\text { Operadores de } \\
\text { instalaciones y máquinas y } \\
\text { ensambladores }\end{array}$ & $\begin{array}{l}\text { Operadores de } \\
\text { instalaciones y maquinaria, } \\
\text { y montadores }\end{array}$ & 06 \\
\hline 9 & Ocupaciones elementales & Ocupaciones elementales & $01,04,10$ \\
\hline 0 & Ocupaciones militares & Ocupaciones militares & 17 \\
\hline
\end{tabular}

\section{Appendix C. Anexo 3}

Resumen de correspondencia entre códigos

\begin{tabular}{ll}
\hline Grupos & Códigos entrevista ZARADEMP \\
\hline Cuello blanco & $08,09,11,12,13,14,15,16$ \\
Cuello azul & $06,07,17$ \\
Labores del hogar & 01,10 \\
Agricultores & $03,04,05$ \\
\hline
\end{tabular}

\section{Bibliografía}

1. Feldman H, Levy AR, Hsiung GY, Peters KR, Donald A, Black SE, et al. A Canadian cohort study of cognitive impairment and related dementias (ACCORD): Study methods and baseline results. Neuroepidemiology. 2003:22:265-74.

2. Ward A, Arrighi HM, Michels S, Cedarbaum JM. Mild cognitive impairment: Disparity of incidence and prevalence estimates. Alzheimers Dement. 2012;8:14.
3. Di Carlo A, Baldereschi M, Amaducci L, Maggi S, Grigoletto F, Scarlato G, et al. Cognitive impairment without dementia in older people: Prevalence, vascular risk factors, impact on disability. The Italian longitudinal study on aging. J Am Geriatr Soc. 2000:48:775.

4. Graham JE, Rockwood K, Beattie BL, Eastwood R, Gauthier S, Tuokko H, et al. Prevalence and severity of cognitive impairment with and without dementia in an elderly population. Lancet. 1997;349:1793.

5. Di Carlo A, Baldereschi M, Lamassa M, Inzitari D, Inzitari M, Scafato E, et al. CIND and $\mathrm{MCI}$ in the Italian elderly: Frequency, vascular risk factors, progression to dementia. Neurology. 2007;68:1909.

6. Palmer K, Wang H, Backman L, Winblad B, Fratiglioni L. Differential evolution of cognitive impairment in nondemented older persons: Results from the Kungsholmen project. Am J Psychiatry. 2002;159:436.

7. Marengoni A, Fratiglioni L, Bandinelli S, Ferrucci L. Socioeconomic status during lifetime and cognitive impairment no-dementia in late life: The Population-Based Aging in the Chianti Area (InCHIANTI) Study. J Alzheimers Dis. 2011;24:559.

8. Marioni RE, Valenzuela MJ, van den Hout A, Brayne C, Matthews FE. Active cognitive lifestyle is associated with positive cognitive health transitions and compression of morbidity from age sixty-five. PloS one. 2012;7:e50940.

9. Dartigues JF, Gagnon M, Letenneur L, Barberger-Gateau P, Commenges D, Evaldre $\mathrm{M}$, et al. Principal lifetime occupation and cognitive impairment in a French elderly cohort (Paquid). Am J Epidemiol. May 1 1992;135:981-8.

10. Jorm AF, Rodgers B, Henderson AS, Korten AE, Jacomb PA, Christensen H, et al. Occupation type as a predictor of cognitive decline and dementia in old age. Age Ageing. 1998;27:477.

11. Cimadevila Álvarez C. Estudio de la relación entre deterioro cognitivo y sintomatología depresiva en la población gallega mayor de 65 años. Univ Santiago de Compostela. 2008.

12. Inzelberg R, Schechtman E, Abuful A, Masarwa M, Mazarib A, Strugatsky R, et al Education effects on cognitive function in a healthy aged Arab population. Int Psychogeriatr. 2007;19:593-603.

13. Helmer C, Letenneur L, Rouch I, Richard-Harston S, Barberger-Gateau P, Fabrigoule C, et al. Occupation during life and risk of dementia in French elderly community residents. J Neurol Neurosurg Psychiatry. 2001;71: 303-9.

14. Valenzuela M, Brayne C, Sachdev P, Wilcock G. Cognitive lifestyle and long-term risk of dementia and survival after diagnosis in a multicenter population-based cohort. Am J Epidemiol. 2011:173:1004.

15. García García F, Sánchez Ayala M, Pérez Martín A. Prevalencia de demencia y de sus subtipos principales en sujetos mayores de 65 años: efecto de la educación y ocupación: Estudio Toledo. Med Clin (Barc). 2001;116:401-7.

16. Fratiglioni L, Winblad B, von Strauss E. Prevention of Alzheimer's disease and dementia. Major findings from the Kungsholmen Project. Physiol Behav. 2007;92(1-2):98-104, 9.

17. Kroger E, Andel R, Lindsay J, Benounissa Z, Verreault R, Laurin D. Is complexity of work associated with risk of dementia? The Canadian Study of Health and Aging. Am J Epidemiol. Apr 1 2008;167:820-30.

18. Anttila T, Helkala EL, Kivipelto M, Hallikainen M, Alhainen $\mathrm{K}$, Heinonen $\mathrm{H}$, et al. Midlife income, occupation, APOE status, and dementia: A population-based study. Neurology. 2002;59:887.

19. Alvarado-Esquivel C, Hernández-Alvarado AB, Tapia-Rodríguez RO, GuerreroIturbe Á, Rodríguez-Corral K, Martínez SE. Prevalence of dementia and Alzheimer's disease in elders of nursing homes and a senior center of Durango City, Mexico. BMC Psychiatry. 2004;4:3.

20. Karp A, Kåreholt I, Oiu C, Bellander T, Winblad B, Fratiglioni L. Relation of education and occupation-based socioeconomic status to incident Alzheimer's disease. Am J Epidemiol. 2004;159:175.

21. He Y, Zhang X, Zhang M. Psychosocial risk factors for Alzheimer's disease. Hong Kong J Psychiatry. 2000;10:2-7.

22. Melendez Moral JC, Mayordomo Rodríguez T, Sales Galán A. Comparación entre ancianos sanos con alta y baja reserva cognitiva y ancianos con deterioro cognitivo. Univ Psychol. 2012;12:73-80.

23. Marioni RE, Proust-Lima C, Amieva H, Brayne C, Matthews FE, Dartigues JF, et al. Cognitive lifestyle jointly predicts longitudinal cognitive decline and mortality risk. Eur J Epidemiol. Mar 2014;29:211-9.

24. Zeki Al Hazzouri A, Haan MN, Osypuk T, Abdou C, Hinton L, Aiello AE. Neighborhood socioeconomic context and cognitive decline among older Mexican Americans: Results from the Sacramento area Latino study on aging. Am J Epidemiol. 2011;174:423-31, 15.

25. Fraser GE, Singh PN, Bennett $H$. Variables associated with cognitive function in elderly California Seventh Day Adventists. Am J Epidemiol. 1996;143: 1181.

26. Lobo A, Saz P, Marcos G, Día J, de-la-Cámara C, Ventura T, et al. The ZARADEMP Project on the incidence, prevalence and risk factors of dementia (and depression) in the elderly community: II. Methods and first results. Eur J Psychiat. 2005; 19:40-54

27. Lobo A, Saz P, Marcos G, Día JL, de-la-Cámara C. The prevalence of dementia and depression in the elderly community in a southern European population. The Zaragoza Study. Arch Gen Psychiatry. 1995;52:497-596.

28. Saz P, Día JL, de la Cámara C, Carreras S, Marcos G, Lobo A. Reliability and validity of the Spanish version of de GSM-AGECAT Package for the assesment of dementia and cognitive disturbances. Int J Geriatr Psychiatry. 1996;11:721-8.

29. Launer LJ, Andersen K, Dewey ME, Letenneur L, Ott A, Amaducci LA, et al., EURODEM Incidence Research Group and Work Groups, European Studies of Dementia. Rates and risk factors for dementia and 
Alzheimer's disease: Results from EURODEM pooled analyses. Neurology. 1999;52:78-84.

30. Lobo A, Saz P, Marcos G, Dia JL, de la Camara C, Ventura T, et al. Revalidation and standardization of the cognition mini-exam (first Spanish version of the MiniMental Status Examination) in the general geriatric population. Med Clin (Barc). 1999; 112:767-74.

31. Folstein MF, Folstein SE, McHugh PR. Mini-mental state. A practical method for grading the cognitive state of patients for the clinician. J Psychiatr Res. 1975;12:189.

32. Perneczky R, Wagenpfeil S, Komossa K, Grimmer T, Diehl J, Kurz A. Mapping scores onto stages: Mini-Mental State Examination and Clinical Dementia Rating. Am J Geriatr Psychiatry. 2006;14:139.
33. Real Decreto $1591 / 2010$, de 26 de noviembre, por el que se aprueba la Clasificación Nacional de Ocupaciones 2011. BOE n. 306 del 17 de diciembre de 2006; Sec. I. pp. 104040-104060.

34. Estructura de la Clasificación Internacional Uniforme de Ocupaciones, 2008 (CIUO-08). Ginebra: Oficina Internacional del Trabajo; 2007.

35. World Health Organization. El estado físico: uso e interpretación de la antropometría: informe de un Comité de Expertos de la OMS. Ginebra: WHO; 1995. p. 387.

36. Hosmer DW, Taber S, Lemeshow S. The importance of assessing the fit of logistic regression models: A case study. Am J Public Health. 1991;81:1630.

37. Nagelkerke NJD. A note on a general definition of the coefficient of determination. Biometrika. 1991;78:691. 